\title{
FRACTURA AISLADA DE PARED MEDIAL DE ÓRBITA*
}

\author{
Drs. David Ibáñez M. ${ }^{1}$, Ruth Rubio C. ${ }^{2}$, Nerea Yanguas B. ${ }^{1}$ \\ 1 Sección de radiodiagnóstico del Hospital Reina Sofía, Tudela, Navarra. \\ 2 CAP Casetas, España. Médico especialista medicina familiar y comunitaria. \\ España.
}

\begin{abstract}
\section{Isolated orbital medial wall fracture}

Background: Orbital floor fractures are a common finding in facial trauma and may be accompanied by medial orbital wall fracture in 7 to $53 \%$ of the cases. Isolated medial wall fractures are rare and usually asymptomatic, being detected as incidental findings. Case report: We report a 75 years old female consulting in the emergency room due to a left orbital trauma. An orbital CAT scan identified a fracture of the medial orbit wall that did not require treatment.
\end{abstract}

Key words: Orbital fracture, medial rectus, ethmoid bone.

\section{Resumen}

Introducción: Las fracturas del suelo de la órbita son habituales en los traumatismos faciales y entre un 7 y un $53 \%$ de los casos se asocian con fracturas de la pared medial. Pero las fracturas aisladas de la pared medial son infrecuentes y la mayoría asintomáticas, siendo un hallazgo habitualmente incidental. Sin embargo, ciertos signos de alarma (limitación de la movilidad del recto medial, siendo habitual que aparezca de forma retardada por isquemia del músculo debido al atrapamiento) pueden plantear una cirugía urgente. Descripción del caso: Presentamos un caso de una paciente de 75 años que acude al Servicio de Urgencias de nuestro hospital remitida por médico de atención continuada por traumatismo sobre órbita izquierda. A la exploración presenta importante hematoma periorbitario y malar, sin alteraciones visuales. En TC orbitaria se identifica fractura de la pared medial de la órbita que, en ausencia de clínica acompañante, no requiere tratamiento alguno.

Palabras clave: Fractura de órbita, recto medial, hueso etmoidal.

\footnotetext{
*Recibido el 17 de junio de 2015 y aceptado para publicación el 14 de agosto de 2015.
}

No existen conflictos de interés.

Correspondencia: Dr. David Ibáñez M. david_ibi@hotmail.com 


\section{Introducción}

Las fracturas del suelo de la órbita son habituales en los traumatismos faciales y entre un 7 y un 53\% de los $\operatorname{casos}^{1,2}$ se asocian con fracturas de la pared medial.

\section{Caso clínico}

Presentamos un caso de una paciente de 75 años que acude al Servicio de Urgencias de nuestro hospital remitida por médico de atención continuada por traumatismo sobre órbita izquierda. A la exploración presenta importante hematoma periorbitario y malar, sin alteraciones visuales. Se realiza tomografía computarizada multidetector (TCMD) identificando una solución de continuidad en la pared medial de órbita izquierda (Figura 1) sin otras fracturas, con engrosamiento y herniación del recto interno (Figura 2, flecha). Es ingresada en el Servicio de Cirugía Maxilofacial para control sin modificación en la motilidad ocular, siendo dada de alta dos días después con tratamiento conservador.

La pared medial de la órbita presenta múltiples tabiques óseos que le dan soporte siendo menos frecuentes sus fracturas que las del suelo orbitario ${ }^{3}$. A menudo son hallazgos incidentales en pacientes asintomáticos en los que se les realiza una tomografía computarizada. Incluso se puede detectar en pacientes que realizan un valsalva tras un traumatismo apareciendo enfisema subcutáneo. Sin embargo, es poco frecuente que se manifiesten con una clara limitación de la movilidad del recto medial, limitándose habitualmente a una paresia del mismo. De hecho es habitual que aparezca de forma retardada por isquemia del músculo debido al atrapamiento ${ }^{4,5}$.

En la exploración clínica se debe evaluar la motilidad ocular en el plano horizontal, y la presencia de enoftalmos (poco frecuente, más habitual si se asocia a fractura del suelo orbitario ${ }^{6}$, hematoma periorbitario o epistaxis). Como clínica atípica se puede dar alteraciones vagales con bradicardia o náuseas/ vómitos por reflejo oculocardiaco. La TCMD es la técnica diagnóstica de elección realizando reconstrucciones coronales adicionales y se debe comparar la distancia de las paredes mediales de ambas órbitas hasta línea media, estando disminuida en el lado de la fractura. La radiografía simple es una técnica obsoleta y de bajo rendimiento diagnóstico ${ }^{6}$.

Las indicaciones de tratamiento quirúrgico son la existencia de enoftalmos clínicamente significativo,

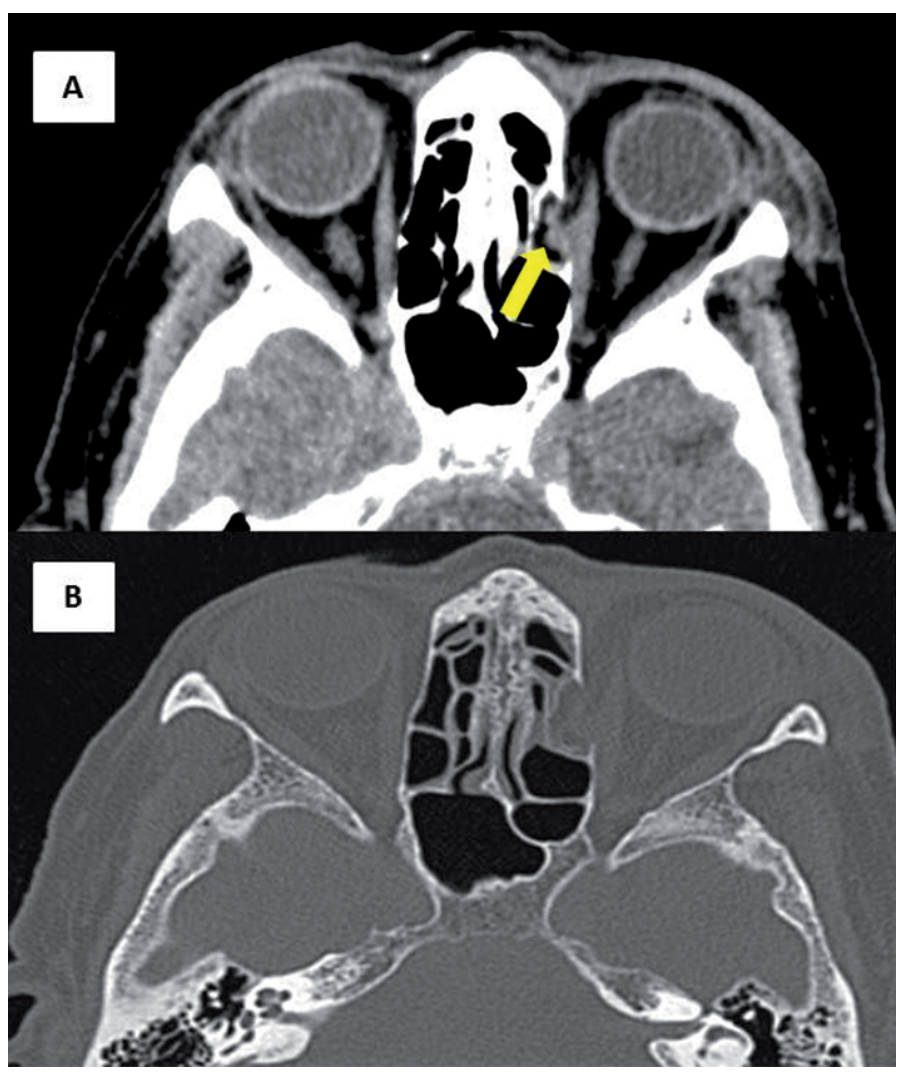

Figura 1. A. Imagen TCMD en plano axial y filtro de partes blandas que muestra solución de continuidad en pared medial de órbita izquierda con engrosamiento y migración del músculo recto medial (flecha). B. Imagen TCMD en plano axial y filtro óseo donde se puede visualizar dicho defecto óseo con mayor precisión. 
o atrapamiento del recto medial en Tc asociado a diplopía, náuseas/vómitos o bradicardia inducida por el movimiento ocular. Los pacientes sin alteración en la motilidad ocular deben ser sometidos a seguimiento para descartar la aparición de enoftalmos diferido.

\section{Referencias}

1. Jank S, Schuchter B, Emshoff R, Strobl H, Koehler J, Nicasi A, et al. Clinical signs of orbital wall fractures as a function of anatomic location. Oral surgery, oral medicine, oral pathology, oral radiology, and endodontics. 2003;96:149-53.

2. Rauch SD. Medial orbital blow-out fracture with entrap- ment. Archives of otolaryngology 1985;111:53-5.

3. Davidson TM, Olesen RM, Nahum AM. Medial orbital wall fracture with rectus entrapment. Archives of otolaryngology. 1975;101:33-5.

4. Brannan PA, Kersten RC, Kulwin DR. Isolated medial orbital wall fractures with medial rectus muscle incarceration. Ophthalmic plastic and reconstructive surgery 2006;22:178-83.

5. Thiagarajah C, Kersten RC. Medial wall fracture: an update. Craniomaxillofacial trauma \& reconstruction 2009;2:135-9.

6. Nolasco FP, Mathog RH. Medial orbital wall fractures: classification and clinical profile. Otolaryngologyhead and neck surgery: official journal of American Academy of Otolaryngology-Head and Neck Surgery. 1995;112:549-56. 